\title{
Anomalous Lithium Adsorption Propensity of Monolayer Carbonaceous Materials: A Density Functional Study
}

\author{
SWATI PANIGRAHI*, DEIVASIGAMANI UMADEVI and G NARAHARI SASTRY* \\ Centre for Molecular Modelling, CSIR-Indian Institute of Chemical Technology, Tarnaka, Hyderabad, \\ Telengana, 500 607, India \\ e-mail: gnsastry@gmail.com; swati.panigrahi06@gmail.com
}

MS received 13 June 2016; revised 30 August 2016; accepted 30 August 2016

\begin{abstract}
Interaction between lithium and carbonaceous materials has gained a lot of importance in lithium battery industry as an important source of energy and storage. The size, dimension, curvature and chirality of the carbonaceous materials are found to be very important factors in controlling the sequential binding of lithium. The propensity of lithium binding to the monolayer carbonaceous materials has been studied using Density functional theory (DFT). Structural and energetical parameters of the complexes have been analyzed through interaction energy, sequential energy, Mulliken population analysis and spin density distribution. Spin density of odd Li doped systems reveals the preferences for addition of further lithium atoms on the surface. Upon analyzing the interaction energy in armchair carbon nanotubes (A-CNTs) and zigzag carbon nanotubes (Z-CNTs), it has been observed that external and internal surfaces of CNTs have contrasting binding preferences for sequential addition of $\mathrm{Li}$ atoms. Internal surface is found to be more feasible site for lithium adsorption than the external surface. This current study provides fundamental understanding of the mechanism of lithium adsorption in lithium battery.
\end{abstract}

Keywords. Energy; adsorption; carbonaceous; sequential; DFT.

\section{Introduction}

There is a high demand for generation of clean and efficient energy sources and Lithium battery is emerging as the most alternative power source in this era. ${ }^{1}$ Lithium battery provides high energy density and a longer life cycle, thus an ideal candidate to replace the conventional battery systems. Lithium battery comprising of graphitic anode, an electrolyte and cathode material mostly comprises of $\mathrm{LiCoO}_{2}$ and it operates in the following principle. During charging, Li ions are released from the cathode and moves towards the anode and intercalated between the graphite layers, while the mechanism is reverse during discharging process. ${ }^{2-4}$ Carbonaceous materials have gained tremendous attention in the development of lithium ion batteries as they can reversibly absorb and release lithium ions at low electrochemical potentials. ${ }^{5-7}$ The reversible capacity of the battery depends on the type of the cathode and anode material. ${ }^{8}$ Graphite has been used as an anode material in many commercial grade lithium ion batteries with a storage capacity of 372 milliamp hours per gram $\left(\mathrm{mAhg}^{-1}\right){ }^{9,10}$ However, recently several studies indicated the employment of graphene and CNT

*For correspondence

Celebrating 100 years of Lewis Chemical Bond as anode material in lithium-ion battery. ${ }^{4}$ The good cyclic performance of the graphene nanosheet as anode material has been studied by Wang et al. ${ }^{11}$ Owing to their unique structures and properties, carbon nanotubes exhibit enhanced lithium storage capacity as compared to graphite. ${ }^{12}$ There are several theoretical reports on interaction of graphene with lithium based on density functional theory. ${ }^{13,14}$ Non-covalent functionalization of carbonaceous materials and their interplay is a topic of outstanding importance in its own right. ${ }^{15-17}$ CNT can be used as a prime candidate for anode material due to its unique physical and chemical properties, structural stability, high rigidity with Young's modulus of the order of $1 \mathrm{TPa}$, and high reversible capacity within the range 300 to $600 \mathrm{mAhg}^{-1}$. ${ }^{4,18}$ It has also been reported that mechanical and chemical treatments to the carbon nanotubes could increase the reversible capacities up to $1000 \mathrm{mAhg}^{-1}{ }^{19,20}$ Since CNT has large surface area with both external and internal surfaces are accessible to the metals, it can be used as a better agent for the storage of lithium in lithium ion battery. Rana et al., through theoretical and experimental studies reported intercalation of lithium on the CNT. ${ }^{21}$ Different morphologies of the carbon nanotubes such as chirality and curvature have been reported to have profound impact on the storage capacities of $\mathrm{Li}^{4}{ }^{4,22}$ 
The curvature and chirality of the carbon nanomaterials influence the binding of metal, hydrocarbons, aromatic aminoacids and nucleobases. ${ }^{23-28}$ Interactions between the alkali metals and carbon based materials have several potential roles. ${ }^{17-29}$ Our recent study reported the role of lithium atoms in reducing the polyaromatic hydrocarbons. ${ }^{30}$ Lithium doping increases the reactivity of the graphene and CNT to a greater extent, ${ }^{31,32}$ also increases the conductivity and hydrogen storage capacity. ${ }^{33,34} \mathrm{~A}$ few reports discuss the possibility where lithium resides inside the CNT. Senami et al. ${ }^{35}$ have studied the interaction of lithium on the $(12,0)$ SWCNT using $a b$ initio quantum studies and observed that the adsorptions of one lithium atom inside the SWCNT is more favorable than that of the outer surface and they also observed that adsorption of multiple lithium atoms tends to destabilize the system. However, they have considered CNT with specific dimension. Udomvech et al., also reported first principles calculations of $\mathrm{Li}$ and $\mathrm{Li}^{+}$adsorption on CNT; however most of their modeled CNT are of unit cell cylinder, ${ }^{36}$ and so has certain limitations. The unique tubular structure of the CNT makes it a promising candidate for confinement of elements and encapsulation of molecules. ${ }^{37}$

Curiosity to dwell into the molecular level details of the mechanism of lithium adsorption poses several questions. What is the Li adsorption capacity of buckybowls and carbon nanotubes? Is it the impact of curvature, chirality and size of carbonaceous materials in general, A-CNT/Z-CNT in particular, on the feasibility for Li bonding? How does the Lithium binding lead to structural and energetical changes in the carbon materials? Although considering a few layers of graphene will provide better storage capacity for lithium, single layer configuration turns out to be the most suitable candidate for safe and stable lithium ion batteries operating at extreme high temperatures. ${ }^{38}$ Monolayer graphene is found to be important in its own right and a very recent study by Ludbrook et al., supports this when they show evidences of superconductivity in lithium decorated monolayer graphene. ${ }^{39}$

Delineating the mechanism of lithium adsorption in monolayer carbonaceous materials is interesting in its own right. This study envisioned for development of new carbon based materials for better lithium storage capacity.

\section{Computational Details}

To analyze the lithium storage capacity on different curved carbonaceous materials, we have modeled a range of carbonaceous materials starting from smaller dimensional buckybowls which represent the fragments of the fullerenes i.e., corranulene (A), sumanene (B) and then extended our work by considering CNTs with various dimensions and chirality (Scheme 1). In all the considered geometries, the valencies of dangling electrons are satisfied by hydrogen atoms. ${ }^{40}$ The standard bond lengths and angles of the C-C, C-H, C-C-C are maintained as $1.421 \AA, 1.009 \AA, 120^{\circ}$ in the parent geometry and their interactions with lithium in all possible orientations were carried out. Lithium can bind to the concave $(\mathrm{V})$ or convex $(\mathrm{X})$ surface of the hub $(\mathrm{H})$ or $\operatorname{rim}(\mathrm{R})$ regions of the bowls. Therefore, all the possible combinations were considered in the present study to find out the favorable adsorption site of lithium atoms. Intercalation of lithium on several modified aromatic compounds leads to the formation of aesthetically beautiful molecules which can have many applications in material sciences. ${ }^{41}$ The armchair and zigzag nanotubes of different dimensions are considered separately to have a clear view of how the absorption of lithium depends on the chirality and dimension of the nanotubes. Arm chair nanotubes such as $(4,4)$, $(5,5),(6,6),(7,7)$ and zigzag nanotubes with dimensions $(8,0),(10,0),(12,0),(14,0)$ are modeled for the present investigation. Since both external and internal surfaces of CNTs are accessible to the metals, ligand, ions etc., we have considered both the surfaces separately in the present study. Throughout the manuscript we have followed the nomenclature as mentioned in Scheme 1.

Lithium atoms are allowed to interact sequentially with the carbonaceous materials considered in the present study and full geometry optimization of all the lithium-carbonaceous complexes have been carried out with B3LYP (Becke, three-parameter, Lee-YangParr) functional in the framework of density functional theory (DFT). Optimization of corannulene and sumanene complexes were carried out through both the 3-21G and 6-31G* level (however, all further calculations are done considering B3LYP/6-31G* optimized geometry), while 3-21G level was used for the optimization of CNTs. All the calculations were performed using Gaussian 09 program. ${ }^{42}$ The change in curvature of corannulene and sumanene upon metal complexation were examined by measuring the bowl depth (BD), which is the calculated as the distance between centroid of the hexagonal ring of the hub $(\mathrm{H})$ region and the plane formed by the carbon atoms of the rim $(\mathrm{R})$ region (as shown in a schematic diagram in Scheme 1).

The interaction energy of the lithum_carbonaceous complexes was calculated by the difference in the total energy of the complex $\left(E_{X Y}^{o p t}\right)$ and energy of 

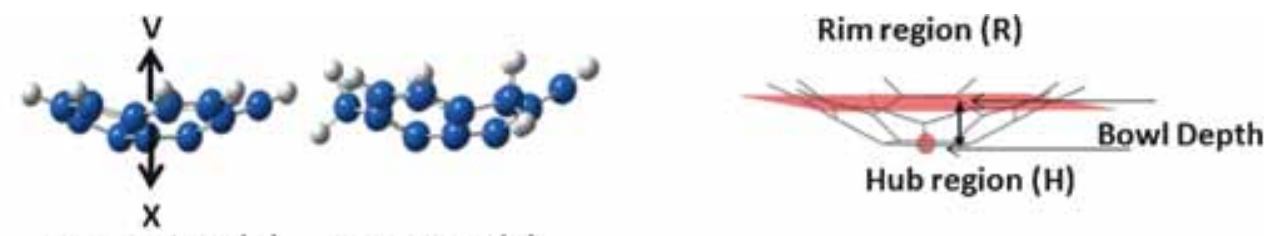

Corannulene (A) Sumanene (B)
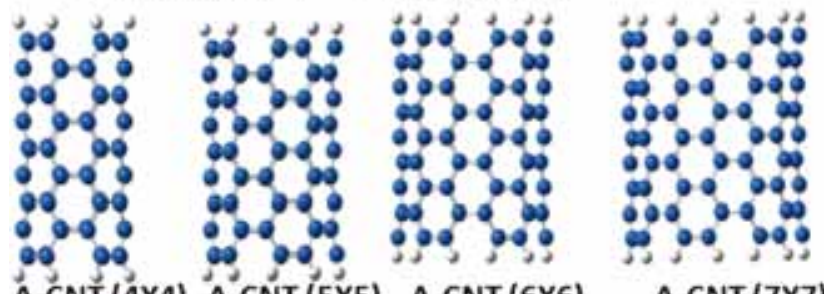

Armchair CNTs

(A-CNTs)

A-CNT (4X4) A-CNT (5X5) A-CNT (6X6)

A-CNT (7X7)


Scheme 1. The model systems corannulene and sumanene (with concave and convex surfaces are denoted as $\mathrm{V}$ and $\mathrm{X}$ ). The carbon nanotubes of different sizes and chirality considered in the present study are shown along with the procedure for calculating the bowl depth (BD).

the monomers $\left(E_{X}^{i s o}\right.$ and $E_{y}^{i s o}$ ) using the following equation

$$
\Delta E=E_{X Y}^{o p t}-E_{X}^{i s o}-E_{y}^{i s o}
$$

We have also calculated sequential interaction energy of the model systems upon adsorption of lithium atoms. The $\Delta E_{2}, \Delta E_{3}, \Delta E_{4}$ are the differences in interaction energy of the model systems upon sequential addition of second, third and fourth lithium atom to the carbonaceous molecules. For example, $\Delta E_{2}$ is calculated using the following equation

$$
\begin{aligned}
\Delta E_{2}= & \Delta E(\text { Carbonaceous_2Li }) \\
& -\Delta E(\text { Carbonaceous_1 } L i)
\end{aligned}
$$

Mulliken population analysis was carried out to have an understanding of electronic distribution of carbonaceous materials upon adsorption of lithium atoms.

\section{Results and Discussion}

We will first discuss about the stable geometry of all the lithium carbonaceous complexes. This will be followed by an elaborate explanation about the structural analysis (Bowl depth), energetic changes (interaction energy, sequential binding energy) and charge transfer (Mulliken population analysis) of these complexes.

\subsection{Model Systems}

The B3LYP/6-31G* optimized geometries of all the buckybowls lithium complexes along with the Li to ring centroid distances (from the nearest hexagonal ring), interaction energy and cumulative Mulliken charges on $\mathrm{Li}$ atom in the optimized geometry are given in Figure 1 . We observed that $\mathrm{Li}$. . ring centroid distance ranges from $1.572 \AA$ to $5.288 \AA$. The interaction energy increases from monolithium to pentalithium adsorption for all the complexes.

We observed that unlike the interaction energy trend, charges on Li do not increase in a linear manner upon sequential adsorption of lithium atoms. No such correlation is observed between the interaction energy and Mulliken charges on lithium atoms for the model systems. We observed a wide variation of Mulliken charges on lithium atoms in case of sumanene $(-0.08$ a.u to 0.302 a.u.), while in corannulene, it ranges between 0.231 to 0.481 a.u. In the optimized form, the neutral lithium acquires fraction of positive charge thereby making the carbonaceous materials anionic in nature. A very peculiar feature was observed for the sumanene molecules, when Li adsorbs towards the convex surface of the bowl, it acquires a fraction of positive charge, however when it interacts through the concave surface of the bowl, the cumulative Mulliken charge comes out to a fraction of negative charge or almost neutral. 

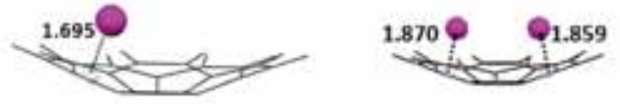

$A 1 \_V,-16.70,0.327$

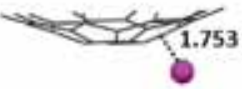

A1_X, -15.54, 0.297

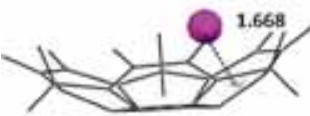

B1 V $,-4.89,0.246$

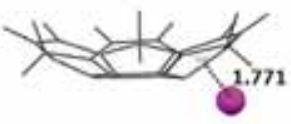

B1_X, -7.83, 0.171
A2_X, - 38.57, 0.481

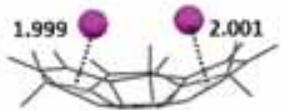

A2_V, -36.29, 0.264

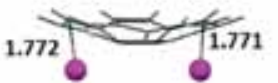

B2 $V,-26.66,0.01$

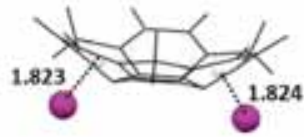

B2 $X,-18.51,0.302$
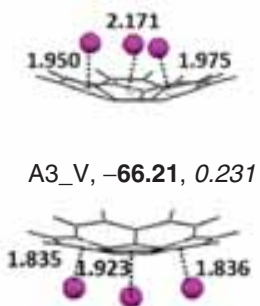

A3_X, -58.68, 0.354
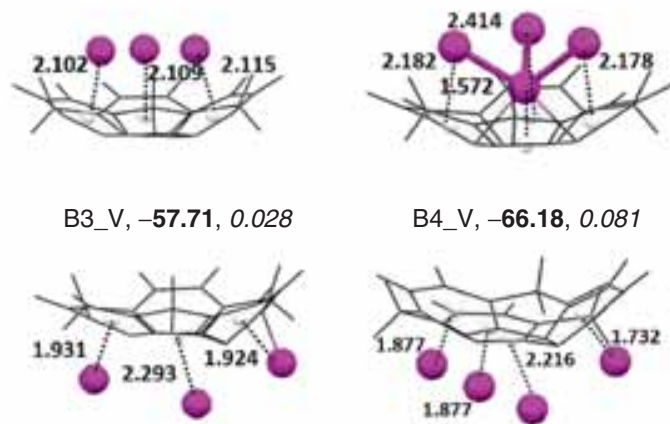

B3_X, $-45.19,0.246$
B4 V $,-66.18,0.081$

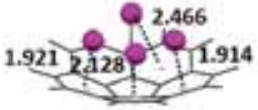

A4_V, -95.62, 0.341
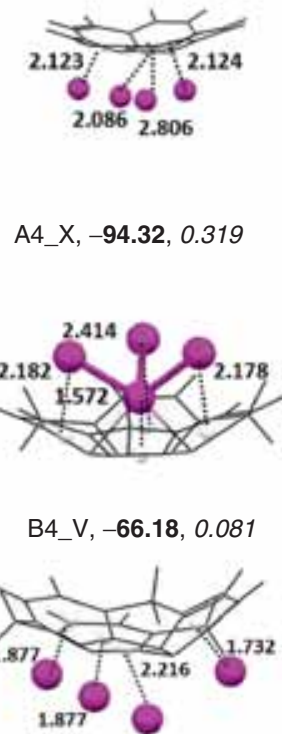

B4_X, -54.94, 0.273
A4_X, -94.32, 0.319

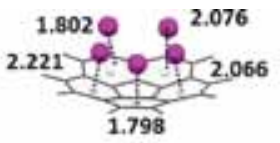

A5_V, -115.22, 0.357


B5_V, -102.35,-0.08

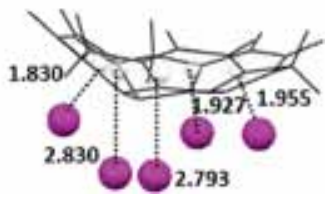

B5_X, -82.05, 0.191

Figure 1. Optimized geometry of the model systems at B3LYP/6-31G* level; Li to ring centroid distances are in $\AA$; interaction energy values in $\mathrm{kcal} / \mathrm{mol}$ (bold); and the cumulative Mulliken charges on Li are in a.u (italics).

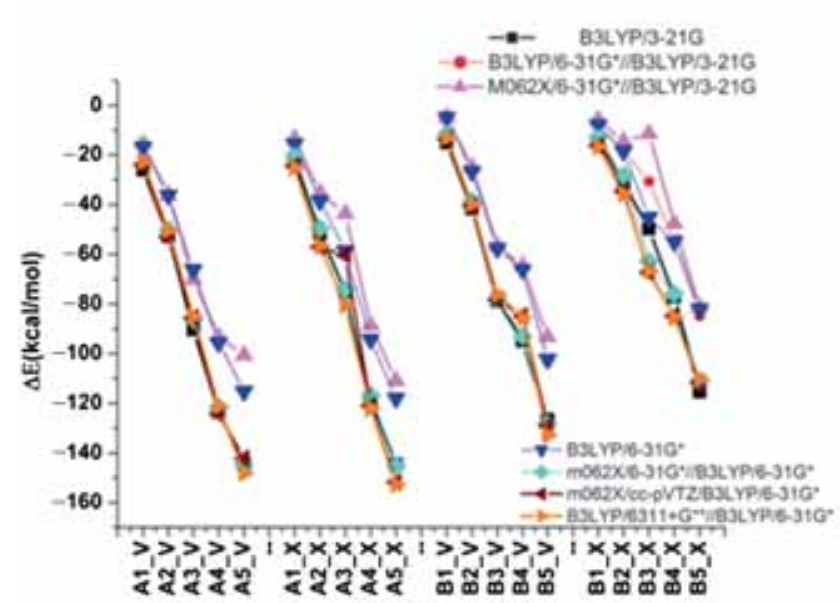

(a)

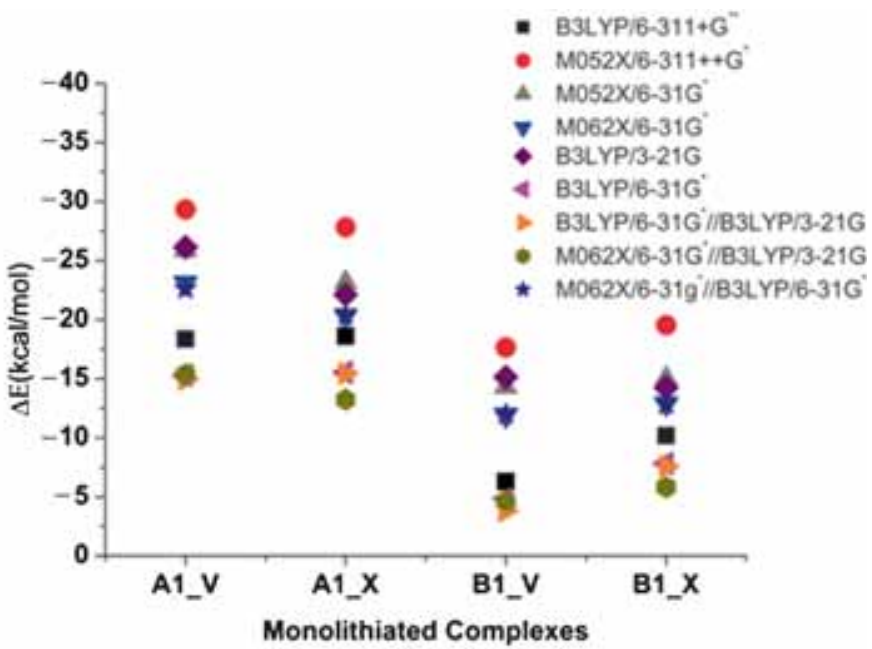

(b)

Figure 2. (a) Interaction energy in $\mathrm{kcal} / \mathrm{mol}$ of corannulene and sumanene complexes upon sequential adsorption of Li atoms by different levels. (b) Standardization of different levels on the monolithiated corannulene and sumanene complexes.

To study the nature of adsorption of lithium in a quantitative manner, we have also calculated single point energy of the complex systems with M062X/ccpVTZ, M062X/6-31G* and B3LYP/6-311+G** level and compared the energy with that of the interaction energy obtained from B3LYP/6-31G* and B3LYP/3$21 \mathrm{G}$ levels. The interaction energies obtained by different methods are presented graphically in Figure 2a.
We observed that interaction energy follows very similar trend by all the methods considered. We have also optimized the monolithiated corannulene and sumanene complexes (with $\mathrm{Li}$ at the concave and convex surface) by a range of quantum chemical levels and presented them graphically in Figure $2 b$, which very clearly proved the reliability of considering the B3LYP/6-31G* level for optimization; also, B3LYP/3-21G level was 
found to be equally good. Interaction energy is found to be maximum for A5_V, A5_X, B5_V and B5_X, which are pentalithium carbonaceous complexes. So we observed that interaction energy increases upon sequential adsorption of lithium atoms in all the carbonaceous materials. It has been reported by several groups that cations prefer to bind to the convex surface of the rim of six-membered ring. ${ }^{43-45}$ However, for neutral lithium adsorption, we observed a different trend. Adsorption of monolithium was found to be more favorable at R-region irrespective of concave or convex surface. Among the monolithiated complexes, corannulene showed higher interaction energy $(-16.70 \mathrm{kcal} / \mathrm{mol}$ (A1_V) and $-15.54 \mathrm{kcal} / \mathrm{mol}\left(\mathrm{A} 1 \_X\right)$ ) than that of the sumanene (-4.89 kcal/mol (B1_V) and $-7.83 \mathrm{kcal} / \mathrm{mol}$ $\left(\mathrm{B} 1 \_\mathrm{X}\right)$ ). Monolithium adsorption was found to be more favorable at the concave surface of corannulene than at the convex surface; however, in sumanene, the order is opposite, convex surface was found to be more favorable adsorption site for lithium. We have also compared the interaction energy of the model systems when $\mathrm{Li}$ is present at the hub region of the buckybowls and the B3LYP/6-31G* optimized geometry are presented in Figure S1 (in Supplementary Information). On comparing the energy and structures, we observed that edges $(\mathrm{R})$ of the buckybowls show strong affinity for adsorption of lithium atoms than the interior region $(\mathrm{H})$. Lithium atoms always show the tendency of moving to the R-region of the sumanene upon optimization when it is placed at the hub region of the convex surface of sumanene. To understand the reason, constrained optimization of the system has been carried out with $\mathrm{C}_{3 \mathrm{v}}$ orientation and energy was obtained as -1.26 $\mathrm{kcal} / \mathrm{mol}$ for the B1_X' geometry (Figure S1 in Supplementary Information), which clearly indicates that hub region of sumanene is energetically unfavorable for the adsorption of Li atoms.

We have calculated the bowl depth (BD) for corannulene and sumanene, which is one way to measure the change in the curvature of the bowls upon lithium adsorption. The variations in $\mathrm{BD}$ of the corannulene and sumanene are presented in Figure S2 (in Supplementary Information). The BDs of isolated corannulene and sumanene are obtained as $0.86 \AA$ and 1.122 $\AA$, respectively. We observed that in corannulene, BD value decreases from the isolated ones upon sequential adsorption of lithium atoms at the concave surface (V), which signifies that adsorption of more number of lithium atoms results in flattening of the corannulene molecule; however, BD does not follow any particular trend when lithium adsorption takes place at the convex (X) surface of corannulene. In case of sumanene, we did not observe any significant alteration in the BD values.
Therefore, we observed that lithium plays an important role in altering the curvature of the buckybowls and the amount of alteration highly depends on the chirality and dimension of the aromatic molecules.

The sequential interaction energy was calculated for all the model systems considered, which gives information about the energy released/adsorbed upon sequential addition of lithium atoms, and given in Table 1. It is very interesting to note that adsorption of third lithium $(-29.92 \mathrm{kcal} / \mathrm{mol})$ and fifth lithium $(-36.17 \mathrm{kcal} / \mathrm{mol})$ are energetically more feasible at the concave site of corannulene (A_V) and sumanene (B_V), respectively; whereas at the convex sites, adsorption of fourth lithium $(-35.64 \mathrm{kcal} / \mathrm{mol})$ in case of corannulene (A_X), and fifth lithium $(-27.10 \mathrm{kcal} / \mathrm{mol})$ in sumanene $\left(B_{-}\right.$X) are more feasible. So, energy varies substantially upon sequential adsorption of lithium atoms. In general, neutral lithium interacts with the carbonaceous material though noncovalent interaction, however, when more than one lithium atoms get absorbed on the surface, additional electrostatic interaction, like $\mathrm{Li}$. . Li interaction comes into play, which may play important role in stabilizing the complex geometry.

On a closure look into the optimized lithium carbonaceous complexes, we observed that second lithium atom always acquires a position which is at least one ring away from that of the position of the first lithium atom on the aromatic ring. Therefore, positioning two lithium atoms on two consecutive five-membered or six-membered ring on same side of the plane of the aromatic molecules is always observed to be repulsive in nature. To understand this, we have analyzed the spin density distribution of the monolithiated complexes. We observed maximum spin density distribution at the edges of the buckybowls. Our primary investigation revealed that positioning of the second lithium depends on the spin density distribution of the monolithiated complex (see Figure S3 in Supplementary Information). Therefore, we can infer that edges of carbonaceous materials provide favorable adsorption sites for lithium atoms.

We have also carried out dilithium interaction through both the plane of corannulene and sumanene with all possible orientations. The B3LYP/6-31G* optimized geometries are given in Figure S4 in Supplementary Information. We observed maximum interaction energy as $-42.66 \mathrm{kcal} / \mathrm{mol}$ for corannulene and -27.31 $\mathrm{kcal} / \mathrm{mol}$ for sumanene. Interaction energy observed in this case was found to be greater than the energy when dilithium interaction takes place either through the concave/convex surface of the corranulene and sumanene molecule. So, dilithium adsorption through both the sides of the plane of the buckybowls is more favorable, 
Table 1. The sequential interaction energy of carbonaceous_lithium complexes $(\mathrm{kcal} / \mathrm{mol})$.

\begin{tabular}{|c|c|c|c|}
\hline Lithium complexes & $\Delta E_{2}$ & $\Delta E_{3}$ & $\Delta E_{4}$ \\
\hline A_V & -19.59 & -29.92 & -29.40 \\
\hline A_X & -23.04 & -20.11 & -35.64 \\
\hline B_V & -21.76 & -31.05 & -8.47 \\
\hline B_X & -10.68 & -26.68 & -9.75 \\
\hline A-CNT $(4,4)$ & -41.36 & -20.09 & -13.97 \\
\hline A-CNT $(5,5)$ & -40.91 & -18.67 & -10.34 \\
\hline A-CNT $(6,6)$ & -39.06 & -16.13 & -11.64 \\
\hline A-CNT $(7,7)$ & -37.65 & -16.68 & -15.90 \\
\hline A-CNT $(6,6){ }^{\#}$ & -44.23 & -46.85 & $-a-$ \\
\hline A-CNT $(7,7)^{\#}$ & -44.40 & -37.20 & $-a-$ \\
\hline Z-CNT $(8,0)$ & -21.51 & -46.72 & -28.37 \\
\hline Z-CNT $(10,0)$ & -37.15 & -46.41 & -32.63 \\
\hline Z-CNT $(12,0)$ & -26.83 & -60.81 & -22.60 \\
\hline Z-CNT $(14,0)$ & -16.70 & -65.54 & -23.50 \\
\hline $\mathrm{Z}-\mathrm{CNT}(10,0){ }^{\#}$ & -41.68 & $-a-$ & $-a-$ \\
\hline Z-CNT $(12,0){ }^{\#}$ & -21.07 & $-a-$ & $-a-$ \\
\hline Z-CNT $(14,0)^{\#}$ & -6.70 & -65.06 & -19.62 \\
\hline
\end{tabular}

Note: For A and B complexes, sequential interaction energy was calculated at B3LYP/6-31G* level and for A-CNT, Z-CNT complexes energy was obtained at B3LYP/3-21G level.

\#Represents addition of Li atom/atoms at the internal surface of the A-CNT and Z-CNT. -a- signifies where no more lithium can be added due to geometrical restriction.

which correlates well with our recently reported observations. ${ }^{27}$

\subsection{Carbon nanotubes}

To study the effect of lithium atom/atoms on higher dimensional structures, we have considered carbon nanotube as a standard model with various dimensions and chirality. Both the armchair (A-CNTs) and zigzag nanotubes (Z-CNTs) were considered. One of the interesting property of the nanotube is that both external and internal surfaces are accessible to other ligands, ions, metals, etc. So we have placed lithium atom/atoms at both the surfaces of A-CNTs and Z-CNTs and studied all the complex systems in a detailed manner. To study the interaction at the external surface, we have considered a number of possible orientations for the adsorption of mono, di, tri and tetra lithium and considered the most stable orientation for further calculations in this paper. To study the interaction of Li atom at internal surface, we have placed $\mathrm{Li}$ atom/atoms unsymmetrically inside the nanotube to allow free movement of the $\mathrm{Li}$. In the initial geometry configuration, $\mathrm{Li}$ atom/atoms are placed in such a way they will not form any covalent bond neither with the sidewall of the nanotube nor with another $\mathrm{Li}$ atom. So, these conditions restrict the complex to have very few orientations of the Li atom/atoms inside the nanotube. For the $(4,4)$ and $(5,5)$ A-CNTs, we can accommodate only single $\mathrm{Li}$ atom, while for $(6,6)$ and $(7,7)$ A-CNTs, we can accommodate up to three $\mathrm{Li}$ atoms inside the CNT. Whereas, one $\mathrm{Li}$ in $(8,0) \mathrm{Z}-\mathrm{CNT}$, up to two $\mathrm{Li}$ in $(10,0)$ and $(12,0) \mathrm{Z}-\mathrm{CNT}$ and up to four $\mathrm{Li}$ in $(14,0) \mathrm{Z}-\mathrm{CNT}$ can be accommodated. The optimized geometry of CNT_Li complexes along with the interaction energy of the complex systems and cumulative Mulliken charges on $\mathrm{Li}$ atoms (with external and internal surfaces of the A-CNT decorated with lithium atoms) are given in Figures S5 and S6 in Supplementary Information, respectively, and the optimized geometry of ZNT_Li complexes (with external and internal faces of the Z-CNT decorated with lithium atoms) are given in Figures S7 and S8 in Supplementary Information, respectively.

The trend in interaction energy of the systems with lithium at the external and internal faces is shown in Figure 3. We observed that interaction energy increases upon sequential adsorption of $\mathrm{Li}$ atoms in both the A-CNT and Z-CNT of varying dimensions. On comparing the interaction energy at the external and internal surfaces of A-CNT and Z-CNT, we observed that $\mathrm{Li}$ atom/atoms is/are found to be stabilized better at the internal surface than that of the external surface, irrespective of the dimension of the A-CNT and $\mathrm{Z}$-CNT considered. So, internal surface is more favorable adsorption site for the lithium atom/atoms in both the A-CNT and Z-CNT. The sequential interaction energy data (Table 1) also reveals that adsorption of second lithium at the external surface is found to be more feasible in all the dimensions of A-CNT, while adsorption of third lithium atom is found to be more 

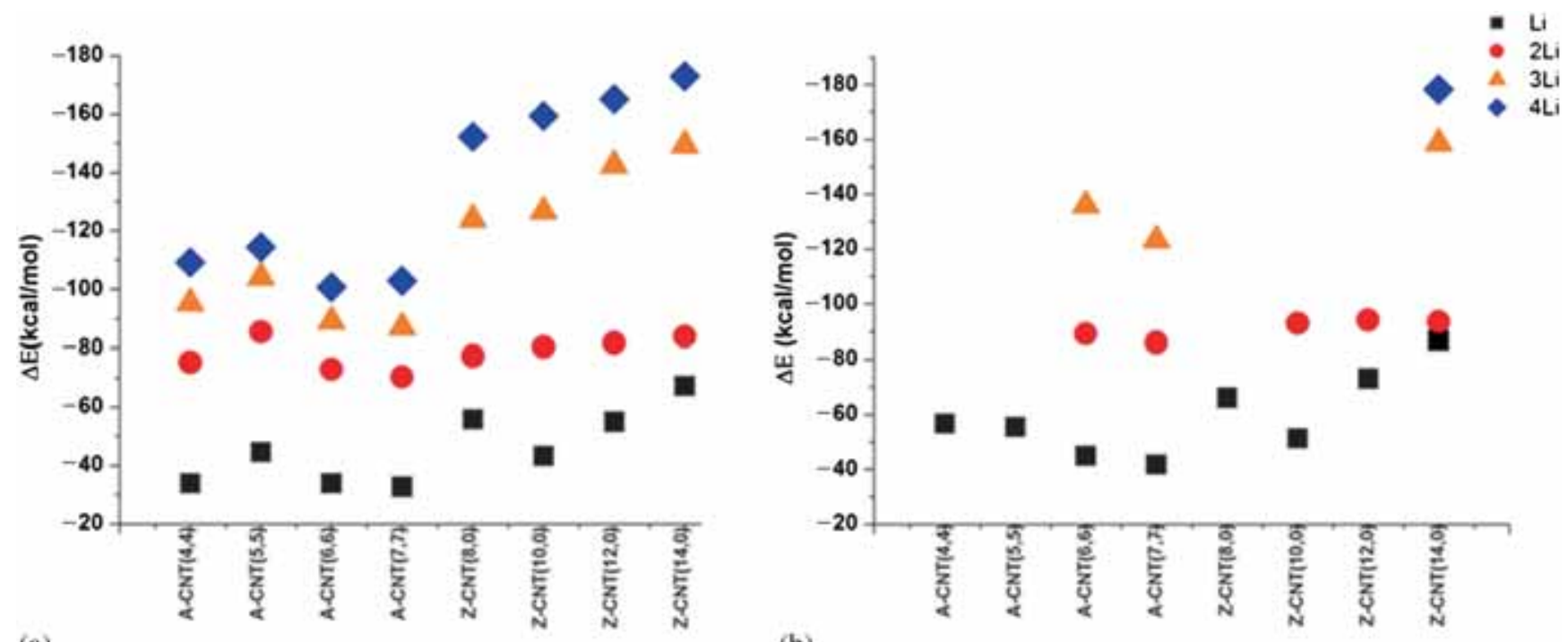

(a)

(b)

Figure 3. Trend in interaction energy ( $\mathrm{kcal} / \mathrm{mol})$ of the Li complexes when sequential Li adsorption takes place: (a) external, and (b) internal surface of the carbon nanotube at B3LYP/3-21G level.

feasible at the external surface of Z-CNT. Mulliken charges on $\mathrm{Li}$ increases upon sequential adsorption of $\mathrm{Li}$ atoms in both A-CNT and Z-CNT. We have also considered the third possibility of adsorption of dilithium atoms in $\mathrm{A}-\mathrm{CNT}$ and $\mathrm{Z}-\mathrm{CNT}$, where $\mathrm{Li}$ atoms interact simultaneously through both the external and internal surfaces of the A-CNT and Z-CNT. We have considered two different orientations for the adsorption of lithium atoms, (i) when lithium atoms interact with the same hexagonal ring, (ii) lithium atoms interact with two different hexagonal rings. The B3LYP/3-21G optimized geometry of the A-CNT and Z-CNT lithium complexes are shown in Figures S9 and S10 in Supplementary Information, respectively. Interaction energy is observed to be maximum for the second condition, when lithium atoms interact through different hexagonal rings, i.e., interaction energy of A-CNT $(4,4) \_2 \mathrm{a}^{\$}, \mathrm{~A}-\mathrm{CNT}(5,5) \_2 \mathrm{a}^{\$}$, A-CNT $(6,6) \_2 \mathrm{a}^{\$}$, A-CNT $(7,7) \_2 \mathrm{a}^{\$}$ are obtained as $-73.28,-78.54,-62.58$, and $-58.26 \mathrm{kcal} / \mathrm{mol}$, respectively. In the same manner, we observed maximum interaction energy for the Z-CNT $(8,0) \_2 \mathrm{a}^{\$}(-68.86$ $\mathrm{kcal} / \mathrm{mol})$, Z-CNT $(10,0) \_2 \mathrm{a}^{\$} \quad(-68.96 \mathrm{kcal} / \mathrm{mol})$, Z-CNT(12,0)_2a $\mathrm{a}^{\$}(-64.45 \mathrm{kcal} / \mathrm{mol})$, Z-CNT(14,0)_2a ${ }^{\$}$ $(-68.84 \mathrm{kcal} / \mathrm{mol})$, where $\mathrm{Li}$ adsorption takes place through different hexagonal rings, as compared to the Z-CNT_2 $b^{\$}$ complexes, where lithium adsorption takes place through the same hexagonal ring.

So, on comparing all the three possibilities of the adsorption of dilithium atoms, such as (i) interaction through the external surface only, (ii) interaction through the internal surface only, and (iii) interaction through both the external and internal surfaces simultaneously, we observed maximum interaction energy when lithium atoms interact through the internal surface of the A-CNT and Z-CNT irrespective of the dimension of A-CNT and Z-CNT. Interaction of lithium atoms through the same hexagonal ring is less feasible. Irrespective of the dimension of the nanotube considered in this present analysis, the interaction energy of the Z-CNT_dilithium complexes follows the trend,

$$
\begin{aligned}
& \text { Z-CNT_2 } 2^{\#}>\text { Z-CNT_2 }>\text { Z-CNT_2a }{ }^{\$}> \\
& \text { Z-CNT_2b }
\end{aligned}
$$

(where the superscript ${ }^{\#}$ symbolizes Li interaction through the internal surface of CNT/ZNT, no superscript represents $\mathrm{Li}$ interaction through the external surface of CNT/ZNT and \$ represents when Li atoms interact simultaneously through the internal and external surface of the CNT/ZNT).

We observed the same trend for the A-CNT complexes also.

\section{Conclusions}

An exhaustive analysis has been carried out to study the lithium adsorption on the carbonaceous materials such as corannulene, sumanene and CNT. In case of corannulene and sumanene, the concave surface $(\mathrm{V})$, convex surface $(\mathrm{X})$, the $\operatorname{rim}(\mathrm{R})$ and hub $(\mathrm{H})$ region were considered separately and in case of carbon nanotube the armchair and zigzag nanotubes were treated differently. We have allowed $\mathrm{Li}$ atoms to interact through both the external and internal surfaces of the A-CNT and Z-CNT. Interaction energy and Mulliken population analysis were carried out to understand the stability of the complex systems. Interaction energy increases 
upon sequential adsorption of $\mathrm{Li}$ atoms in all the carbonaceous materials. By measuring the bowl depth, we found that Li plays important role in altering the curvature of the buckybowls. Adsorption of more Li atoms generally flattens the corannulene. Spin density is found to be mostly localized at the edges of the buckybowls, which favors the adsorption of second lithium atom consecutively. Adsorption of dilithium through both sides of the plane of corannulene and sumanene were found to be most favorable. Upon comparing the favorable site of adsorption of lithium to the nanotube, we observed that internal surface of the nanotube is the more favorable site for $\mathrm{Li}$ in both the armchair and zigzag nanotube, as compared to external surface.

Thus, our study provides in depth understanding on the adsorption of $\mathrm{Li}$ on buckybowls and carbon nanotubes with various size, dimension and chirality. Understanding the sequential interaction energies of $\mathrm{Li}$ atoms with carbon nanotubes will provide useful insights about their storage capacity, which in turn may provide important platform in designing new carbonaceous anode materials in Li batteries.

\section{Acknowledgements}

We thank CSIR-12 ${ }^{\text {th }}$ five year projects and TCOF fellowships for the support. GNS thanks DST for the award of J C Bose National fellowship. SP acknowledges the support of DST Young Scientist scheme.

\section{Supplementary Information (SI)}

Figures S1 to S10 are available in the Supplementary Information at www.ias.ac.in/chemsci.

\section{References}

1. Scrosati B and Garche J 2010 J. Power Sources 195 2419

2. Van der Ven A, Bhattacharya J and Bela A 2013 Acc. Chem. Res. 461216

3. Xiong Z, Yun Y and Jin H-J 2013 Materials 61138

4. de las Casas C and Li W 2012 J. Power Sources 20874

5. Landi B J, Ganter M J, Cress C D, DiLeo R A and Raffaelle R P 2009 Energy Environ. Sci. 2638

6. Marschilok A, Lee C-Y, Subramanian A, Takeuchi K J and Takeuchi E S 2011 Energy Environ. Sci. 42943

7. Bruce P G, Scrosati B and Tarascon J M 2008 Angew. Chem. Int. Ed. 472930

8. Ritchie A G 2001 J. Power Sources 961

9. Dahn J R 1991 Phys. Rev. B 449170

10. Tarascon J M and Armand M 2001 Nature 414359

11. Wang G, Shen X, Yao J and Park J 2009 Carbon 472049

12. Shimoda H, Gao B, Tang X P, Kleinhammes A, Fleming L, Wu Y and Zhou O 2001 Phys. Rev. Lett. 88015502

13. Garay-Tapia A, Romero A H and Barone V $2012 \mathrm{~J}$. Chem. Theory Comput. 81064
14. Khantha M, Cordero N, Molina L, Alonso J and Girifalco L 2004 Phys. Rev. B 70125422

15. Umadevi D, Panigrahi S and Sastry G N 2014 Acc. Chem. Res. 472574

16. Mahadevi A S and Sastry G N 2016 Chem. Rev. 116 2775

17. Umadevi D and Sastry G N 2011 J. Phys. Chem. C 115 9656

18. Morris R S, Dixon B G, Gennett T, Raffaelle R and Heben M J 2004 J. Power Sources 138277

19. Chen Y, Li X, Park K, Song J, Hong J, Zhou L, Mai Y-W, Huang H and Goodenough J B 2013 J. Am. Chem. Soc. 13516280

20. Lim H, Shin H S, Shin H-J and Choi H C 2008 J. Am. Chem. Soc. 1302160

21. Rana K, Kucukayan-Dogu G, Sen H S, Boothroyd C, Gulseren O and Bengu E 2012 J. Phys. Chem. C 116 11364

22. Yang Z, Ren J, Zhang Z, Chen X, Guan G, Qiu L, Zhang $\mathrm{Y}$ and Peng H 2015 Chem. Rev. 1155159

23. Umadevi D and Sastry G N 2012 Chem. Phys. Lett. 549 39

24. Umadevi D and Sastry G N 2013 Chem. Phys. Chem. 14 2570

25. Panigrahi S, Bhattacharya A, Banerjee $S$ and Bhattacharyya D 2012 J. Phys. Chem. C 1164374

26. Umadevi D and Sastry G N 2011 J. Phys. Chem. Lett. 2 1572

27. Umadevi D and Sastry G N 2014 Front. Chem. 275

28. Umadevi D and Sastry G N 2014 Curr. Sci. 1061224

29. Rytkönen K, Akola J and Manninen M 2004 Phys. Rev. B 69205404

30. Panigrahi S and Sastry G N 2014 RSC Adv. 414557

31. Denis P A 2011 J. Phys. Chem. C 11520282

32. Denis P A 2011 J. Phys. Chem. C 11513392

33. Kolmann S J, Chan B and Jordan M J 2008 Chem. Phys. Lett. 467126

34. Cabria I, López M and Alonso J A 2005 J. Chem. Phys. 123204721

35. Senami M, Ikeda Y, Fukushima A and Tachibana A 2011 AIP Adv. 1042106

36. Udomvech A, Kerdcharoen T and Osotchan T 2005 Chem. Phys. Lett. 406161

37. Pan X and Bao X 2011 Acc. Chem. Res. 44553

38. Chan Y and Hill J M 2011 Nanoscale Res. Lett. 6203

39. Ludbrook B M, Levy G, Nigge P, Zonno M, Schneider M, Dvorak D J, Veenstra C N, Zhdanovich S, Wong D, Dosanjh P, Straßer C, Stöhr A, Forti S, Ast C R, Starke U and Damascelli A 2015 Proc. Natl. Acad. Sci. U. S. A. 11211795

40. Koskinen P, Malola S and Häkkinen H 2008 Phys. Rev. Lett. 101115502

41. Saá J M and Yañez M 2009 Chem. Eur. J. 153123

42. Frisch M J, Trucks G W, Schlegel H B, Scuseria G E, Robb M A, Cheeseman J R, Scalmani G, Barone V, Mennucci B., Petersson G A, Nakatsuji H, Caricato M, Li X, Hratchian H P, Izmaylov A F, Bloino J, Zheng G, Sonnenberg J L, Hada M, Ehara M, Toyota K, Fukuda R, Hasegawa J, Ishida M, Nakajima T, Honda Y, Kitao O, Nakai H, Vreven T, Montgomery J A Jr, Peralta J E, Ogliaro F, Bearpark M, Heyd J J, 
Brothers E, Kudin K N, Staroverov V N, Kobayashi R, Normand J, Raghavachari K, Rendell A, Burant J C, Iyengar S S, Tomasi J, Cossi M, Rega N, Millam J M, Klene M, Knox J E, Cross J B, Bakken V, Adamo C, Jaramillo J, Gomperts R, Stratmann R E, Yazyev O, Austin A J, Cammi R, Pomelli C, Ochterski J W, Martin R L, Morokuma K, Zakrzewski V G, Voth G A, Salvador P, Dannenberg J J, Dapprich S, Daniels A D, Farkas Ö,
Foresman J B, Ortiz J V, Cioslowski J and Fox D J 2009 (Gaussian Inc.: Wallingford CT)

43. Mahadevi A S and Sastry G N 2013 Chem. Rev. 113 2100

44. Priyakumar U and Sastry G N 2003 Tetrahedron Lett. 44 6043

45. Vijay D, Sakurai H, Subramanian V and Sastry G N 2012 Phys. Chem. Chem. Phys. 143057 